\title{
En Europe...
}

\section{lasi-NG : un concentré d'innovations technologiques pour l'étude de l'atmosphère terrestre}

Les sondeurs infrarouges hyperspectraux embarqués à bord de satellites permettent le suivi, de jour comme de nuit, sur terre comme sur mer, d'un grand nombre de variables climatiques, thermodynamiques et de composants chimiques de l'atmosphère. La mission Iasi, réalisée par le Cnes en collaboration avec Eumetsat dans le cadre de son programme EPS-Metop de satellites météorologiques européens en orbite polaire, a mis en évidence la gamme très vaste des composés chimiques et climatiques qu'il était possible de mesurer ou de détecter grâce aux observations dans l'infrarouge thermique $\left(\mathrm{H}_{2} \mathrm{O}, \mathrm{CO}_{2}, \mathrm{CO}, \mathrm{CH}_{4}, \mathrm{O}_{3}, \mathrm{SO}_{2}\right.$, $\mathrm{NH}_{3}$, aérosols, etc.) (Phulpin et al., 2011 ; Hilton et al., 2012). Iasi a permis de dresser des cartes d'espèces qui n'avaient jamais pu être observées auparavant à l'échelle globale et depuis l'espace, et assure un suivi en temps quasi réel de gaz clefs pour l'étude du climat et de la chimie atmosphérique. $\mathrm{La}$ nécessité pour la météorologie opérationnelle de disposer de sondeurs infrarouges performants a également été illustrée par Iasi : son impact sur les performances des prévisions numériques du temps de différents centres (MétéoFrance, Centre européen pour les prévisions météorologiques à moyen terme, Met Office, etc.) est le plus important parmi les instruments spatiaux aujourd'hui disponibles pris individuellement.

Les sondeurs infrarouges hyperspectraux apportent ainsi une forte contribution à trois domaines qui deviennent de plus en plus liés : 1) la prévision numérique du temps aux échelles globale ou régionale, à court et moyen terme ; 2) la composition atmosphérique, y compris la pollution ; 3) le climat.

Le programme satellitaire EPS-SG d'Eumetsat est destiné à prendre la suite du programme EPS sur la période allant de 2020 à 2035 et au-delà. Dans ce cadre, le Cnes a initié le développement de la mission Iasi-nouvelle génération (Iasi-NG), proposée par les scientifiques français en 2009. Trois exemplaires de Iasi-NG seront embarqués sur les trois plateformes successives Metop-SG-A avec une durée d'opération de 7 ans chacune (Clerbaux et Crevoisier, 2013). La présence en orbite d'au moins un satellite sera assurée en permanence sur cette période.

\section{Objectifs de la mission: plus de résolution verticale et meilleure précision}

Les grands programmes internationaux ont fait apparaître le besoin crucial d'observations atmosphériques plus précises afin de mieux caractériser les processus, de valider les modèles et d'assurer un suivi sur le long terme de la composition thermodynamique et chimique de l'atmosphère. En accord avec ces besoins, les trois objectifs scientifiques de la mission Iasi-NG sont : 1) l'amélioration de la résolution verticale par rapport à Iasi qui permettra notamment de mieux observer l'atmosphère proche de la surface (épisodes de pollution, inversions de température), ainsi que dans la région de la tropopause (afin de mieux appréhender les échanges entre la troposphère et la stratosphère, telle l'intrusion d'ozone stratosphérique, qui influencent à la fois le climat et la chimie de l'atmosphère) ; 2) l'amélioration de la précision des estimations des différentes variables, ainsi que la détection de nouveaux composants atmosphériques ; 3) la continuité du programme Iasi pour le suivi sur le long terme des variables climatiques essentielles.

Afin de répondre à ces objectifs, l'instrument Iasi-NG a été conçu pour avoir « plus de résolution spectrale et moins de bruit instrumental » que Iasi (au moins un facteur 2 sur ces deux caractéristiques), tout en assurant la continuité de la mission Iasi. En effet, les études menées par le groupe scientifique de la mission (Crevoisier et al., 2013) ont montré : 1) qu'une meilleure résolution spectrale et un meilleur bruit radiométrique sont synonymes d'une meilleure couverture verticale, en particulier dans les basses couches de l'atmosphère ; 2) qu'ils permettent aussi une amélioration de la précision des mesures (meilleur rapport signal sur bruit, moins d'interférences entre les signatures spectrales des molécules) ; 3) que les seuils de détection pour les espèces faiblement absorbantes sont également améliorés.

\section{Le défi majeur: la compensation de champ}

Iasi et Iasi-NG sont des interféromètres à transformée de Fourier qui donnent accès au spectre de rayonnement infrarouge émis par le système Terreatmosphère, à partir duquel sont retrouvées les différentes variables atmosphériques et de surface. Dans un tel instrument, le faisceau incident de rayonnement monochromatique est divisé par une lame semi-réfléchissante, appelée séparatrice, en deux parties approximativement égales, qui parcourent des trajets optiques de longueurs différentes avant de se combiner à nouveau sur la séparatrice. Cette recombinaison de deux ondes cohérentes déphasées donne lieu à un phénomène d'interférences. L'interférogramme est la mesure de ces interférences, que l'on fait varier avec la différence de marche (différence de longueur de chemin optique entre les deux bras de l'interféromètre) grâce au miroir mobile. L'interférogramme est ensuite converti en spectre infrarouge par transformation de Fourier.

Dans la pratique, cette transformation n'est pas parfaite. Elle est discrète, bruitée et limitée par la différence de marche maximale permise par le mécanisme et par les effets de champ. Ces derniers viennent du fait que, pour un pixel étendu vu sous un grand angle d'observation, l'instrument reçoit des rayonnements multiples sous un angle d'incidence suffisamment élevé par rapport à son axe pour entraîner une altération du spectre mesuré. Or, l'amélioration de la précision radiométrique de Iasi-NG d'au moins un facteur 2 par rapport à Iasi nécessite une augmentation du champ d'observation de l'instrument, et l'amélioration de la résolution spectrale nécessite un doublement de la différence de marche maximale à parcourir. La conception de Iasi-NG a donc requis une rupture technologique permettant la compensation des effets de champ.

\section{Une solution instrumentale innovante fondée sur l'interféromètre de Mertz}

La solution proposée par l'industriel Airbus Defence and Space, qui a été retenue pour la réalisation de l'instrument, s'appuie sur une architecture optique qui repose sur le principe conçu 

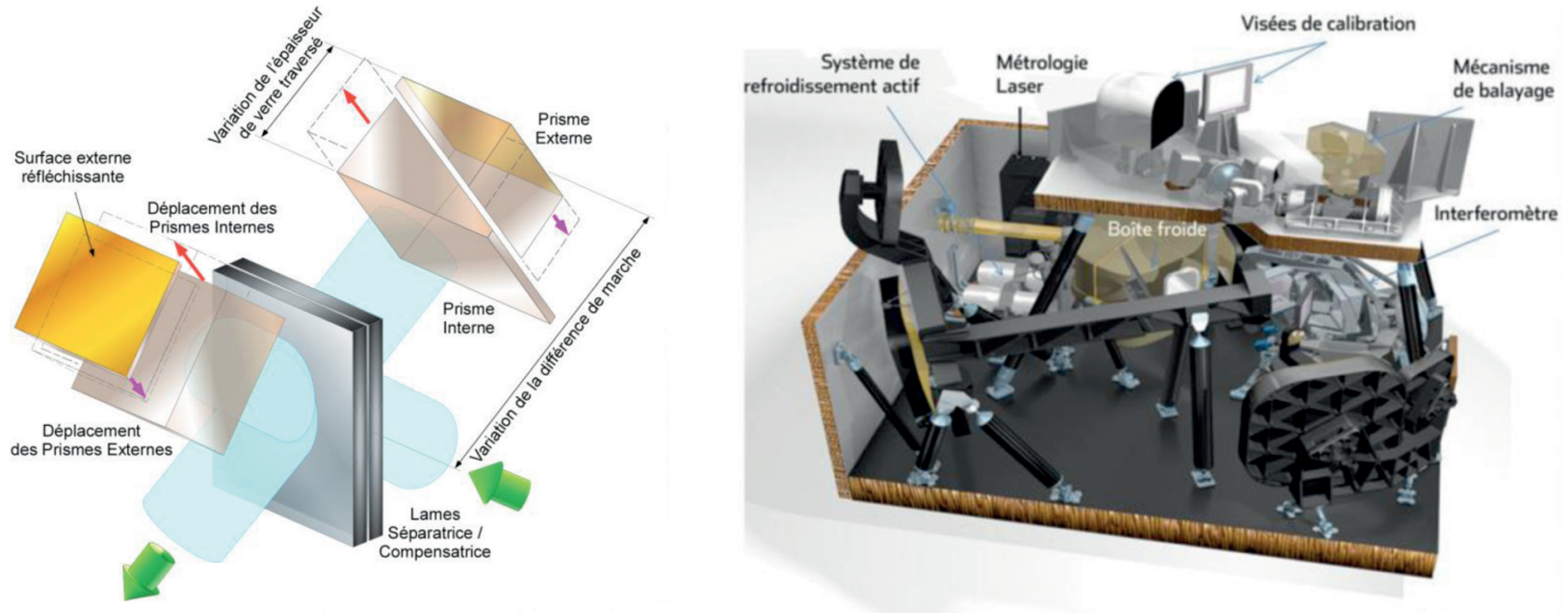

Figure 1. À gauche : principe du cœur interférométrique de lasi-NG. À droite : vue des différents éléments de l'instrument.

par Mertz et Connes dans les années 1960. Le cœur interférométrique de l'instrument (figure 1) est composé de trois éléments : 1) une lame séparatrice/compensatrice qui divise et reconstruit le faisceau optique ; 2) des miroirs mobiles qui introduisent la différence de marche entre les faisceaux ; 3) des prismes triangulaires se translatant selon leurs hypoténuses qui font varier l'épaisseur du matériau que doivent traverser les faisceaux afin de compenser les effets de champ. Les faces arrières des prismes extérieurs sont recouvertes d'une pellicule réfléchissante et jouent le rôle de miroirs mobiles. Dans la configuration de référence, le matériau retenu est le bromure de potassium $(\mathrm{KBr})$ qui permet d'assurer une excellente transmission optique sur l'ensemble du spectre infrarouge.

Le mécanisme de cœur interférométrique est particulièrement novateur (Luitot et al., 2013) et s'appuie sur un mécanisme à double balancier (Dual Swing Mechanism) breveté par le Cnes et Airbus Defence and Space. Ce

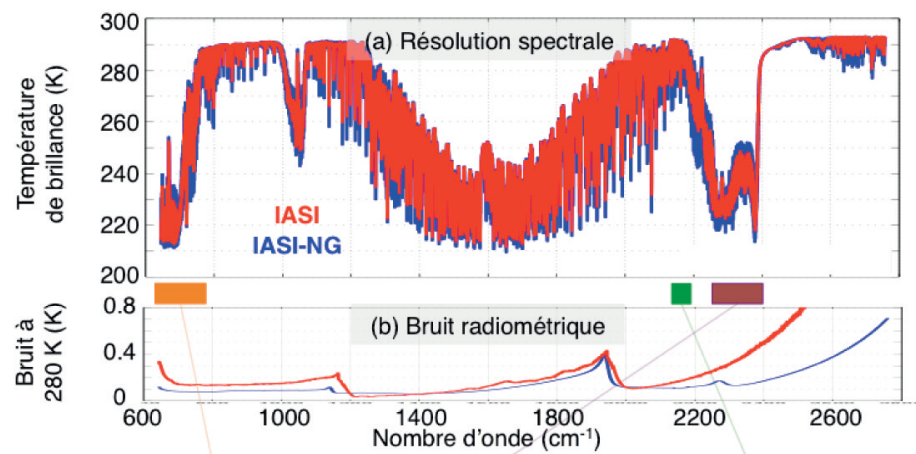

(c) Estimation de la température

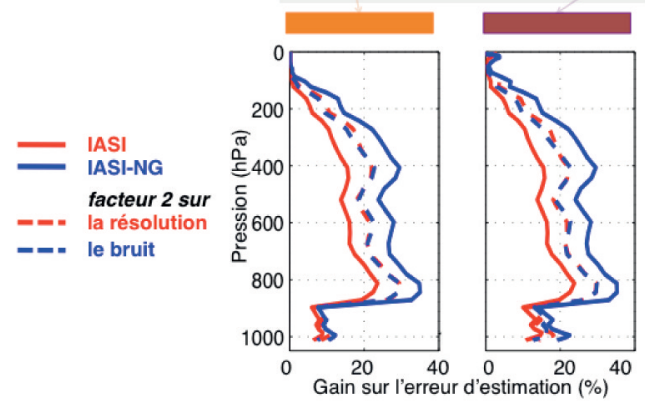

(d) Estimation du $\mathrm{CO}$

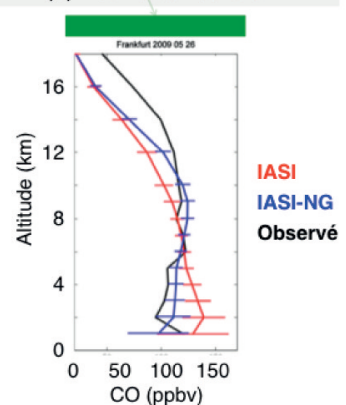

Figure 2. Caractéristiques spectrales et radiométriques de lasi (rouge) et lasi-NG (bleu) et leur impact sur la restitution de variables atmosphériques. (a) Spectre des températures de brillance pour une atmosphère tropicale à la résolution spectrale de lasi et de lasi-NG. (b) Bruits radiométriques de lasi et lasi-NG à une température de référence de $280 \mathrm{~K}$. (c) Gain sur l'erreur d'estimation par rapport à l'a priori (\%) des profils atmosphériques de température à partir de deux bandes spectrales (645$770 \mathrm{~cm}^{-1}$ et 2250-2420 $\mathrm{cm}^{-1}$ ). En pointillé, gain lorsque seule la résolution spectrale ou le bruit est amélioré. (d) Comparaison des restitutions de monoxyde de carbone (CO) à partir de lasi et lasi-NG et comparaison avec le vrai profil.

mécanisme, qui assure la translation synchrone entre les prismes intérieurs et extérieurs, réalise en un seul mouvement la compensation de champ et la variation de la différence de marche nécessaire pour obtenir des interférences.

La chaîne de détection s'apparente à Iasi dans son principe, même si les technologies utilisées sont différentes. En particulier, la performance radiométrique visée nécessite un refroidissement des détecteurs à une température de $75 \mathrm{~K}$ par un système de refroidissement actif. Enfin, un système de métrologie avancée fondé sur au moins quatre lasers sera embarqué (figure 1).

Les caractéristiques géométriques de l'instrument seront proches de celles de Iasi, avec une tache au sol au nadir de 12 $\mathrm{km}$. Sur le même intervalle spectral que Iasi (nombres d'onde 645 à $2760 \mathrm{~cm}^{-1}$ ), la résolution spectrale de $0,25 \mathrm{~cm}^{-1}$ (deux fois mieux que Iasi) sera assurée par une différence de marche maximale de $4 \mathrm{~cm}$ et conduira à une meilleure résolution des raies d'absorption des constituants atmosphériques (le renforcement de leur signature est bien visible sur le spectre de la figure 2a). Le bruit radiométrique (figure $2 b$ ) sera divisé par un facteur d'au moins 2 par rapport à Iasi ; en particulier, le bruit sera nettement amélioré dans les courtes longueurs d'onde, une région spectrale particulièrement importante pour sonder l'atmosphère proche de la surface.

\section{Les principales avancées scientifiques attendues}

L'amélioration simultanée de la résolution spectrale et du bruit radiométrique, rendue possible par le concept instrumental retenu, permettra de répondre 
aux objectifs scientifiques de la mission décrits ci-dessus. Le tableau 1 détaille les principales améliorations en termes de résolution verticale et de précision de la restitution qu'apportera Iasi-NG sur les principales variables thermodynamiques, chimiques et climatiques. Par exemple, en termes de température, la résolution verticale sera doublée et la précision améliorée d'environ $25 \%$. Comme le montre la figure $2 \mathrm{c}$, c'est bien l'amélioration simultanée de la résolution spectrale et du bruit radiométrique qui permettra ce gain significatif. La meilleure couverture des basses couches de l'atmosphère est bien visible sur la précision attendue sur l'estimation de nombreuses variables atmosphériques dont par exemple le monoxyde de carbone, l'un des gaz réactifs majeurs étudiés avec les sondeurs infrarouges hyperspectraux, pour lequel la restitution des profils atmosphériques sera nettement améliorée près de la surface, notamment dans les cas de forte pollution (figure 2d).

Plus qu'une simple continuation de Iasi, Iasi-NG sera un atout pour les sciences de l'atmosphère, dans les domaines de la prévision numérique du temps, de l'étude de la composition atmosphérique et du climat reposant sur une profonde innovation technique et technologique.

Tableau 1. Comparaison des résolutions verticales (Rés. vert.), exprimées en termes de degrés de liberté (DOF) qui indiquent le nombre d'informations indépendantes que l'on peut avoir sur la verticale, et des erreurs de restitution (\%) disponibles avec lasi et attendues avec lasi-NG. CL : couche limite. Tropo : troposphère. Strato : stratosphère.

\begin{tabular}{|c|c|c|c|c|c|}
\hline Objectif & lasi & & lasi-I & & \\
\hline Thermodynamique & Rés. vert. (DOF) & Erreur $(\%)$ & Rés. vert. (DOF) & Erreur (\%) & Apport de la « NG » \\
\hline Température & 6 & $\sim 0,6 \mathrm{~K}$ & 12 & $\sim 0,45 \mathrm{~K}$ & Amélioration d'un facteur 2 sur la résolution verticale \\
\hline $\mathrm{H}_{2} \mathrm{O}$ & $5-6$ & $\sim 13 \%$ & $6-7$ & $\sim 10 \%$ & Amélioration d'un facteur 1,5 sur l'erreur \\
\hline Chimie & Rés. vert. (DOF) & Erreur $(\%)$ & Rés. vert. (DOF) & Erreur (\%) & Apport de la "NG » \\
\hline $\mathrm{O}_{3}$ & $3-4$ & $\begin{array}{l}\text { CL: } 60 \% \\
\text { Tropo : } 11 \%\end{array}$ & $4-5$ & $\begin{array}{l}C L: 40 \% \\
\text { Tropo : } 8 \%\end{array}$ & $\begin{array}{l}\text { Amélioration de l'information CL et séparation } \\
\text { Tropo/Strato }\end{array}$ \\
\hline $\mathrm{CO}$ & $1-2$ & $\begin{array}{l}\text { CL: } 16 \% \\
\text { Tropo : } 8 \%\end{array}$ & $2-3$ & $\begin{array}{l}C L: 10 \% \\
\text { Tropo: } 6 \%\end{array}$ & Amélioration de l'information CL \\
\hline $\mathrm{NH}_{3}, \mathrm{HCOOH}, \mathrm{CH}_{3} \mathrm{OH}, \mathrm{C}_{2} \mathrm{H}_{4}$ & Détecté & : & Mesuré & - & Mesure et non simplement détection \\
\hline $\mathrm{SO}_{2}$-volcans, $\mathrm{H}_{2} \mathrm{~S}$-volcans & Mesuré si > 2 DU & - & Mesuré si > 1 DU & - & Altitude du panache estimée \\
\hline Climat & Rés. vert. (DOF) & Erreur $(\%)$ & Rés. vert. (DOF) & Erreur $(\%)$ & Apport de la « NG» \\
\hline $\mathrm{CO}_{2}$ & 1 ou moins & $\sim 1 \%$ & $1-2$ & $<1 \%$ & Accès à la moyenne troposphère \\
\hline $\mathrm{CH}_{4}$ & 1 ou moins & $3 \%$ & $1-2$ & & Moins d'interférences avec $\mathrm{H}_{2} \mathrm{O}$ \\
\hline $\mathrm{N}_{2} \mathrm{O}$ & Détecté & i- & Mesuré & - & \\
\hline Aérosols & Poussières & & & & $\begin{array}{l}\text { Mesures d'autres types d'aérosols } \\
\text { (ex: feux de biomasse) }\end{array}$ \\
\hline
\end{tabular}

Cyril Crevoisier Laboratoire de météorologie dynamique, IPSL, CNRS, École polytechnique, Palaiseau, France

Cathy Clerbaux Laboratoire atmosphères, milieux, observations spatiales, IPSL, CNRS, Université Pierre-et-Marie-Curie, Paris, France

Vincent Guidard

CNRM-Game, Météo-France, CNRS, Toulouse, France

Éric Pequignot

Centre national d'études spatiales, Toulouse, France

Frédérick Pasternak

Airbus Defence and Space, Toulouse, France

\footnotetext{
Clerbaux C., Crevoisier C., 2013. New directions: infrared remote sensing of the troposphere from satellite: less, but better. Atmos. Env., 72, 24-26, doi:10.1016/j.atmosenv.2013.01.057

Crevoisier C., Clerbaux C., Guidard V., Phulpin T., Armante R., Barret B., Camy-Peyret C., Chaboureau J.-P., Coheur P.-F., Crépeau L., Dufour G., Labonnote L., Lavanant L., Hadji-Lazaro J., Herbin H., Jacquinet-Husson N., Payan S., Péquignot E., Pierangelo C., Sellitto P., Stubenrauch C., 2013. Towards IASI-New Generation (IASI-NG): impact of improved spectral resolution and radiometric noise on the retrieval of thermodynamic, chemistry and climate variables. Atmos. Meas. Tech. Discuss., 6, 11215-11277.

Hilton F. et al., 2012. Hyperspectral Earth Observation from IASI: Five years of accomplishments. Bull. Am. Meteorol. Soc., 93, 347-370.

Luitot C., Boyadjian J., Buil C., Pasternak F., 2013. Optical architecture of the new generation infrared atmospheric sounder interferometer (IASI-NG). Proc. SPIE 8841, Current Developments in Lens Design and Optical Engineering XIV, 88410M, doi:10.1117/12.2025236.

Phulpin T., Camy-Peyret C., Taylor J., Clerbaux C., Coheur P., Crevoisier C., Edwards D., Gambacorta A., Guidard V., Hilton F., Jacquinet N., Knuteson R., Lavanant L., McNally T., Matricardi M., Revercomb H., Serio C., Strow L., Schlüssel P., Klaes D., Larigauderie C., 2011. Les résultats exceptionnels de Iasi, sondeur atmosphérique hyperspectral de Metop. La Météorologie, 72, 19-30.
} 